\title{
Workers of the primitively eusocial wasp Ropalidia marginata do not perceive their queen across a wire mesh partition
}

\author{
A. Sumana - Sujata A. Deshpande - Anindita Bhadra • \\ Raghavendra Gadagkar
}

Received: 18 October 2006/Accepted: 14 April 2007/Published online: 7 June 2007

(C) Japan Ethological Society and Springer 2007

\begin{abstract}
Queens of primitively eusocial wasps generally have active and behaviourally dominant queens who use physical aggression to suppress worker reproduction. Although a Ropalidia marginata queen is strikingly docile and behaviourally non-dominant, she is completely successful in maintaining reproductive monopoly. $R$. marginata queens must achieve such reproductive monopoly by some means other than overt physical aggression. Upon loss or removal of the queen, one of the workers (referred to as the potential queen) becomes extremely aggressive and will eventually go on to become the next queen of the colony, if the original queen is not returned. The fact that potential queens are not discernible in the presence of the queen but become obvious within minutes after removal of the queen raises the question of how workers in general and the potential queens in particular, perceive the presence or absence of their queens. Here, we have conducted experiments in which we separate half of the workers from their queen by a wire mesh screen and study their behavioural response to such separation. We demonstrate that the presence of the queen is not perceived across the wire mesh screen, which suggests that if the queen uses a pheromone to signal her presence, then that pheromone is not very volatile.
\end{abstract}

\footnotetext{
A. Sumana $\cdot$ S. A. Deshpande $\cdot$ A. Bhadra .

R. Gadagkar $(\bowtie)$

Centre for Ecological Sciences, Indian Institute of Science,

Bangalore 560012, India

e-mail: ragh@ces.iisc.ernet.in

R. Gadagkar

Evolutionary and Organismal Biology Unit,

Jawaharlal Nehru Centre for Advanced Scientific Research,

Jakkur, Bangalore 560064, India
}

Keywords Ropalidia marginata $\cdot$ Primitively eusocial wasp - Potential queen - Dominance behaviour . Non-volatile pheromone

\section{Introduction}

Primitively eusocial wasps are characterized by relatively small colonies $(<100$ individuals) and absence of morphological divergence between queens and workers. By these criteria Ropalidia marginata may be classified as primitively eusocial. But, unlike other primitively eusocial wasps which have active, behaviourally dominant queens who use physical aggression to suppress worker reproduction (West-Eberhard 1969, 1977; Chandrashekara and Gadagkar 1991; Fletcher and Ross 1985; Reeve 1991), an $R$. marginata queen is strikingly docile and behaviourally non-dominant (Premnath et al. 1996; Kardile and Gadagkar 2002; Sumana and Gadagkar 2003). It must be emphasized however that the behaviourally inactive and non-dominant, docile queens of $R$. marginata are completely successful in suppressing reproduction of their workers and maintaining their reproductive monopoly (Gadagkar 2001). It seems clear therefore that $R$. marginata queens must achieve such reproductive monopoly by some means other than overt physical aggression in the form of dominance behaviour (Premnath et al. 1996; Gadagkar 2001; Sumana and Gadagkar 2001).

Upon loss or removal of the queen, one of the workers will become extremely aggressive and will eventually go on to become the next (docile) queen of the colony, if the original queen does not return. We therefore label this individual as the potential queen (Premnath et al. 1996; Deshpande et al. 2006). The fact that potential queens are not discernible in the presence of the queen but become 
obvious within minutes after removal of the queen raises the question of how workers in general and the potential queens in particular, perceive the presence or absence of their queens. Such perception cannot be mediated through dominance or other behavioural interactions because of the extreme paucity of such interactions relative to the time taken to perceive the absence of the queen (Sumana and Gadagkar 2003; Bhadra et al. 2007). Visual recognition of the queen seems unlikely due to the absence of morphological caste differentiation and of obvious cuticular markings as in some species of Polistes (Tibbetts 2002; Tibbetts and Dale 2004).

Thus, the most attractive hypothesis in the context of $R$. marginata is that regulation of worker reproduction by established queens and the perception of the queens by workers is mediated by pheromones. Any attempts to localize and identify possible pheromones would be greatly facilitated by the knowledge of whether they might be volatile or non-volatile compounds. If the queen pheromone is volatile, her presence should be perceived by the workers across a wire mesh partition, and if the queen pheromone is non-volatile, the queen should not be perceived across the mesh and therefore a potential queen should become obvious on the queen-less side of the mesh.

Here, we have therefore conducted experiments in which we separate half of the workers from their queen by a wire mesh screen and study their behavioural response to such separation. We demonstrate that the presence of the queen is not perceived across the wire mesh screen suggesting that if the queen uses a pheromone to signal her presence, then that pheromone is not very volatile.

\section{Methods}

Post-emergence nests of $R$. marginata were located in various nesting sites in Bangalore $\left(13^{\circ} 00^{\prime} \mathrm{N}, 77^{\circ} 32^{\prime} \mathrm{E}\right)$, Mysore $\left(12^{\circ} 25^{\prime} \mathrm{N}, 76^{\circ} 50^{\prime} \mathrm{E}\right)$ and Mudumalai $\left(11^{\circ} 34^{\prime} \mathrm{N}\right.$, $76^{\circ} 38^{\prime} \mathrm{E}$ ), and transplanted to the Vespiary at the Centre for Ecological Sciences, Indian Institute of Science, Bangalore (Gadagkar 2001). All individuals in each colony were uniquely marked with small spots of quick drying paints of different colours for observations. Behavioural observations were carried out on the colonies using randomly intermingled all occurrences sessions and instantaneous scans, each lasting for $5 \mathrm{~min}$, and followed by a 1-min break. All acts of dominance behaviours were recorded and used to compute the frequency of dominance behaviour (for a description of the sampling methodology and of dominance behaviours, see Gadagkar 2001). The queen was identified by observing her egg-laying behaviour.

Twenty-two colonies of $R$. marginata, with 8-37 adult wasps, were used between September 2000 and March
2003 for these experiments. All colonies were maintained in closed cages of $30 \times 30 \times 30 \mathrm{~cm}$ and provided with ad libitum food, water, honey and softwood as a source of fibre for nest construction. On the first day of the experiment, the unmanipulated colony was observed between 0900 and 1,800 for $6 \mathrm{~h}$ in two observation blocks of $3 \mathrm{~h}$ each, to obtain 20-30 scans and 30-40 all occurrences sessions. On the next day, all the adults were collected, a detailed map of the nest was constructed and the nest was cut into approximately equal halves. Both fragments of the colony were then transplanted, one on each side of a wire mesh partition $(2 \times 2 \mathrm{~mm})$, in a closed cage. All the adults were released on one of the two fragments, such that one fragment had the queen and a randomly chosen set of half the workers (hereafter referred to as the queen-right side), while the other had only the other half of the workers (hereafter referred to as the queen-less side). Unlike in the case of other experiments where the orphaned workers were not allowed to interact with their queens (Downing and Jeanne 1985; Gobin et al. 1999; Dietemann et al. 2005; Liebig et al. 1999), the individuals in this case could interact through the mesh partition. We confirmed that the queen-right and queen-less nest fragments were not significantly different from each other in the numbers of the eggs, larvae and pupae present in them (Wilcoxon matched-pairs signed-ranks test; $T=5.0-10.5$ and $P>0.05$ ). Five to $6 \mathrm{~h}$ of behavioural observations were conducted between 1,130 and $1,900 \mathrm{~h}$ yielding $35-40$ all occurrences and 12-20 scans. The same observer observed both the queen-right and queen-less fragments simultaneously.

In 12 of the 22 experiments, observations were continued on the third day, after moving the queen from the queenright side to the queen-less side, so that the queen-less fragment of day 2 became the queen-right fragment on day 3 and vice-versa. Observations were carried out as on day 2 . In eight of the experiments, the queen was not exchanged on day 3. Instead, both nest fragments were maintained in the same closed cage for 8-10 days, with the mesh partition intact. At the end of this period, all the adult wasps were collected and frozen at $-20^{\circ} \mathrm{C}$ until they were dissected. After the adults were removed, the queen-right and queenless fragments were examined and a nest map was constructed. These nest maps were used to identify new eggs that were laid after the separation of the two nest fragments on day 2. A total of 151 wasps were dissected, their ovaries were measured and an index of ovarian development was calculated, as described in Chandrashekara and Gadagkar (1991).

To see if the queen-less fragments behaved like queenless colonies, we compared our results with those obtained from observations of queen-less colonies in separate experiments as described in Sumana and Gadagkar (2003). All statistical comparisons between different classes of 
individuals (queens, potential queens, etc.) within each colony were made using the Wilcoxon matched-pairs signed-ranks test, and comparisons across colonies were made using the Mann-Whitney $U$ test. Statistical comparisons were made using the software StatistiXL version 1.5 .

\section{Results}

When $R$. marginata colonies were split into two halves separated by a wire mesh screen, workers in the queen-less fragment behaved very differently from those in the queenright fragment; the latter behaved no differently from workers in the unmanipulated colony. The rate of dominance behaviour in the queen-less fragment was significantly greater than that in the queen-right fragment or that in the undivided colony. Such an increase in the level of dominance behaviour is also seen in colonies from which the queen is entirely removed (queen removal experiments, see Materials and methods). The rate of dominance behaviour in the queen-less fragment was not significantly different from that in the queen-less colonies of queen removal experiments (Fig. 1a, b). In addition to being statistically significant, the increase in the level of dominance behaviour in queen-less fragments and queen-less colonies was rather dramatic. In the mesh experiments, the rate of dominance behaviour in queen-less fragments increased 5.8-fold compared to the unmanipulated colony on day one, and 15.4-fold (calculated for 19 nests, because the three remaining nests had 0 value for dominance behaviour in the queen-right fragment) compared to queen-right fragments. This is even more dramatic than the 3.9-fold increase in the rate of dominance behaviour in queen-less colonies compared to queen-right colonies.

\section{Potential queen}

Just as potential queens were recognizable in all queen removal experiments, so were they recognizable in all mesh experiments in the queen-less fragments, but never in the queen-right fragments. The rate of dominance behaviour of the potential queens in queen-less fragments was significantly greater than that of the queen in the queen-right fragment or that of the same individual before the nest was divided. This is similar to the rate of dominance behaviour of potential queens in queen removal experiments, which was significantly greater than that of the same individual before removal of the queen (Fig. 2a, b). Potential queens increased their dominance behaviour 33.9-fold in the mesh experiment and 16.7-fold in the queen removal experiment, as compared with their own behaviour before separation from/removal of, the queen. The queens in most colonies

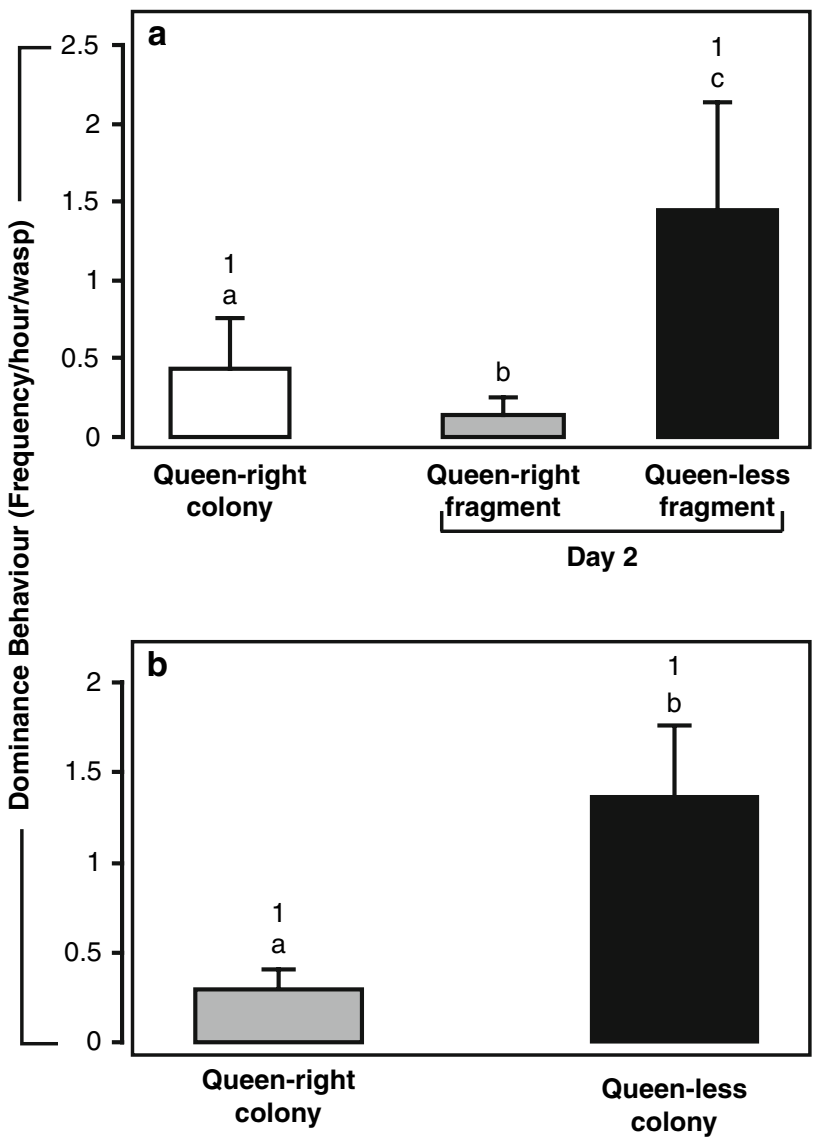

Fig. 1 a Mesh experiment: mean and standard deviation of the frequency per hour per wasp of dominance behaviour in the unmanipulated colony (day 1), the queen-right and queen-less fragments (day 2) ( $n=22$ colonies). b Queen removal experiment: mean and standard deviation of the frequency per hour per wasp of dominance behaviour in the unmanipulated colony (day 1) and the queen-less colony (day 2) $(n=12$ colonies). Within-experiment comparisons are by Wilcoxon matched-pairs signed-ranks test and significantly different values $(P<\alpha$, where $\alpha$ is set to 0.0167 after Bonferroni correction) are indicated using different letters. Between experiment comparisons are by Mann-Whitney $U$ test and significantly different bars $(P<0.05)$ are indicated by different numbers

showed no dominance behaviour, so the fold increase of the potential queen's dominance as compared to the queen could not be calculated.

\section{Queen exchange}

In 12 of the mesh experiments, we moved the queen on day 3 , from the side in which she was on day 2 , to the opposite side. Thus, the queen-less side of day 2 became the queenright side of day 3 . Now the rate of dominance behaviour in the new queen-right side came down significantly and became comparable to that of the queen-right fragment of day 2 (Fig. 3). Besides, in the new queen-right side, the potential queen (PQ 1) evident on day 2 reduced her level of aggression to $12.36 \%$ of her level on the previous day 


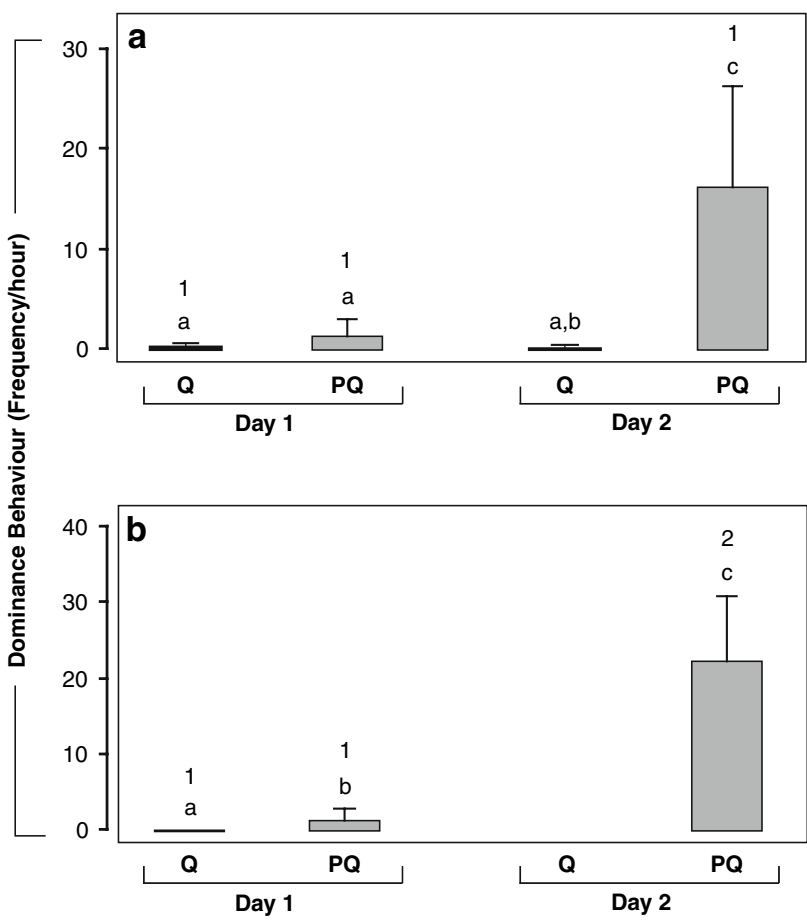

Fig. 2 a Mesh experiment: mean and standard deviation of the frequency per hour of dominance behaviour of the queen and potential queen in the unmanipulated colony (day 1 ), the queen-right and queen-less fragments (day 2) $(n=22$ colonies). b Queen removal experiment: mean and standard deviation of the frequency per hour of dominance behaviour shown by the queen and potential queen on day 1 (unmanipulated colony) and potential queen on day 2 (after queen removal) ( $n=12$ colonies). Within-experiment comparisons are by Wilcoxon matched-pairs signed-ranks test and significantly different values $(P<\alpha$, where $\alpha$ is set to 0.0083 after Bonferroni correction for the mesh experiments and 0.0167 for the queen removal experiments) are indicated using different letters. Between experiment comparisons are as in Fig. 1

and she became indistinguishable from herself on day 1 (Fig. 4). Conversely, the rate of dominance behaviour in the new queen-less side increased significantly and became comparable to that of the queen-less fragment on day 2 (Fig. 3). On day 3, a new potential queen (PQ 2) with high levels of dominance behaviour became evident in the new queen-less fragment; the rate of her dominance behaviour increased significantly as compared to herself on day 1 and 2 (Fig. 4).

\section{Ovarian condition}

In all eight experiments where the queen was not exchanged and where the divided nests were maintained for at least 8 days after the division of the nest, new eggs were seen to have been laid in both queen-right and queen-less fragments. Dissections revealed that the potential queens in the queen-less fragments had developed their ovaries to become indistinguishable from the queens. In spite of the

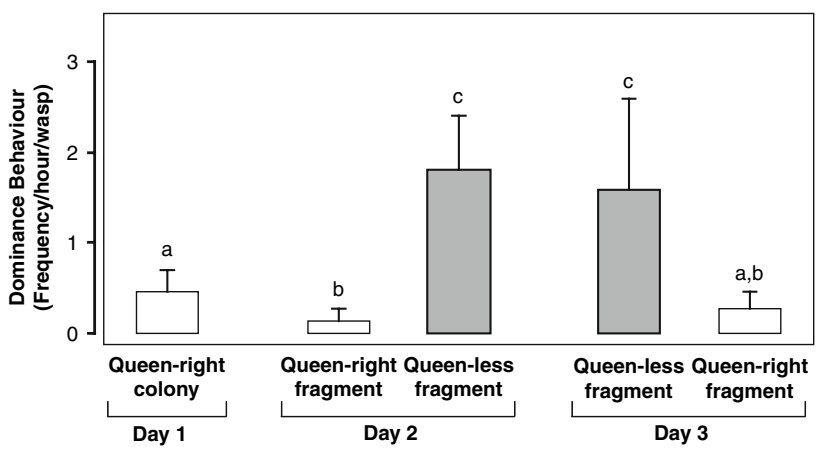

Fig. 3 Mean and standard deviation of the frequency per hour per wasp of dominance behaviour in the unmanipulated colony (day 1), the queen-right and queen-less fragments (day 2) and in the new queen-less and queen-right fragments after the queen was exchanged (day 3 ) in the mesh experiments ( $n=12$ colonies). Comparisons are by Wilcoxon matched-pairs signed-ranks test and significantly different values $(P<\alpha$, where $\alpha$ is set to 0.005 after Bonferroni correction) are indicated using different letters

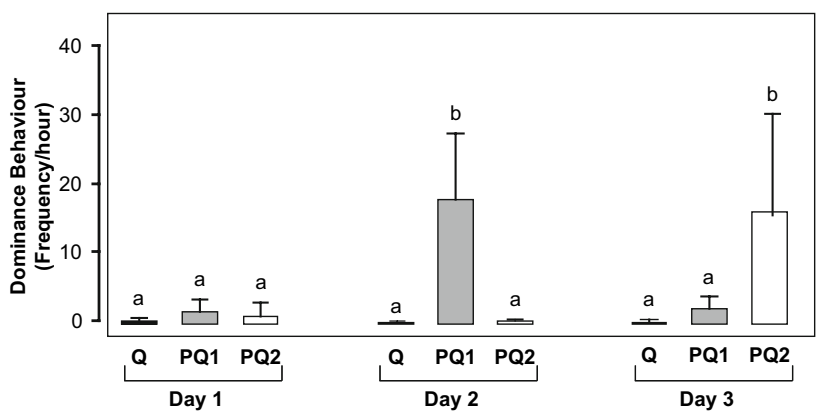

Fig. 4 Mean and standard deviation of the frequency per hour of dominance behaviour of the queen, potential queen 1 (PQ1) and potential queen 2 (PQ2) on day 1 (unmanipulated colony), day 2 (after dividing the colony) and day 3 (after exchanging the queen) $(n=12$ colonies). Comparisons are by Wilcoxon matched-pairs signed-ranks test and significantly different values $(P<\alpha$, where $\alpha$ is set to 0.0023 after Bonferroni correction) are indicated using different letters

high levels of aggression directed by the potential queen to the workers on the queen-less fragment, the ovarian development of an average worker in the queen-less fragment was significantly greater compared to an average worker in the queen-right fragment (Fig. 5).

\section{Discussion}

Because the presence of the queen is not perceived across a wire mesh screen, we conclude that if the queen is using a pheromone to communicate her presence to the workers then the pheromone must be relatively non-volatile. In normal colonies, workers could perceive the queen pheromone either through physical interaction with her or perhaps the queen might apply the pheromone to the nest. 


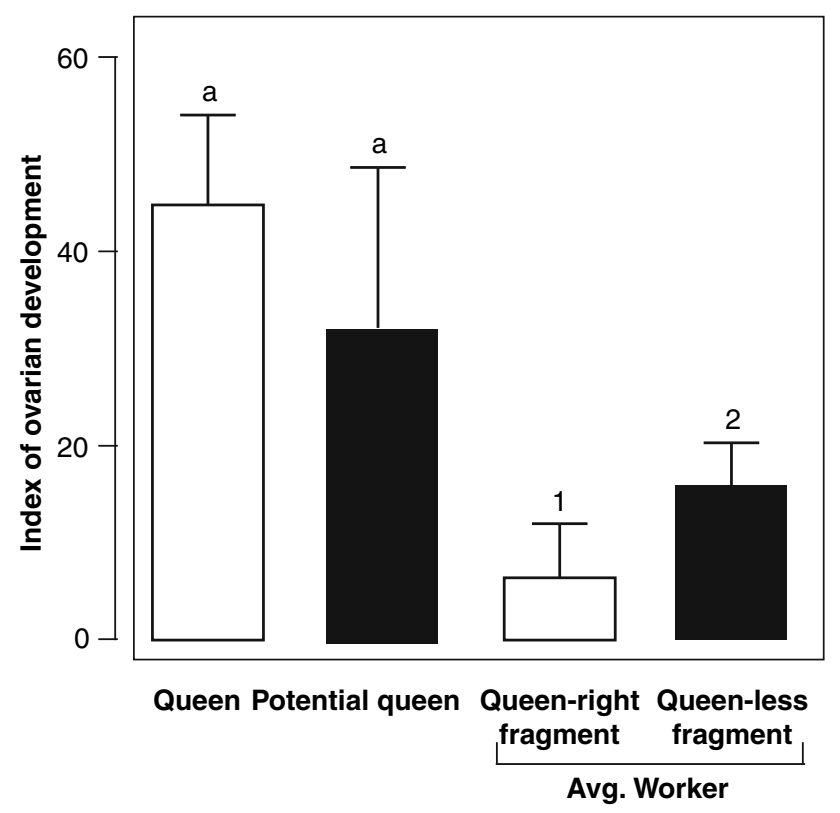

Fig. 5 Mean and standard deviation of the index of ovarian development (see methods) of the queen, potential queen and the workers of the queen-right and queen-less fragments of the mesh experiment ( $n=8$ colonies). Comparisons are between the queen and the potential queen (same letters) and between the average workers on the queen-right and queen-less fragments (different numbers) using Wilcoxon matched-pairs signed-ranks test $(P<\alpha$, where $\alpha$ is set to 0.025 after Bonferroni corrections)

If physical interactions are required for perception, the pheromone might be contained in the cuticular hydrocarbon profile of the queen. This would be reminiscent of the postulates made for queen recognition in social wasps such as Polistes and queen and gamergate recognition in some ponerine ants (Turillazzi et al. 2004; Sledge et al. 2001a, b; de Biseau et al. 2004; Endler et al. 2006; Hannonen et al. 2002; Heinze et al. 2002; Monnin et al. 1998; Tsuji et al. 1999; Liebig et al. 2000; Cuvillier-Hot et al. 2001; D'Ettore et al. 2004; Dietemann et al. 2003). However, we have now demonstrated that physical interactions, direct or indirect (through relay with other workers), are inadequate to explain the transfer of a pheromonal or other signal from the queen to the potential queen. This conclusion is based on a mismatch between the rates of direct and indirect interactions of the queen and potential queen on the one hand, and the time taken by the potential queen to perceive the absence of the queen, on the other (Bhadra et al. 2007). If physical interactions are not required for workers to perceive the queen, the pheromone might be a glandular secretion. However, since it is not very volatile, we speculate that it might be applied to the nest rather than simply released in the air. This speculation is supported by our observation that the queen of $R$. marginata frequently rubs her abdomen on the nest. Data on the rates of such rub abdomen behaviour of the queen (once in $23 \mathrm{~min}$, on average) are consistent with the possibility that the queen applies her pheromone to the nest with the rub abdomen behaviour (Bhadra et al. 2007). It may be noted that the rub abdomen behaviour shown by the $R$. marginata queen is reminiscent of the abdominal stroking behaviour reported for Polistes queens, and which is believed to be used for applying recognition odors on the nest (Dani et al. 1992; Van Hooser et al. 2002). Since the queen pheromone in $R$. marginata may be non-volatile, the use of proteinaceous pheromones (Klobuchar and Deslippe 2002; Turillazzi et al. 2006) is a possibility that deviates from the usual CHC-based pheromones commonly believed to be used in other species. Besides, CHC's do not appear to function as fertility signals in Ropalidia opifex (Dapporto et al. 2006).

The fact that the potential queen is unable to suppress ovarian development in the workers on her side of the mesh, as much as the queen can do on her side, in spite of the very high levels of aggression displayed by her, further strengthens our claim that the queen uses a pheromone rather than physical aggression to regulate/suppress worker reproduction.

Acknowledgments This work was supported by grants from the Department of Science and Technology, Department of Biotechnology and the Ministry of Environment and Forests, Government of India (to Raghavendra Gadagkar), a Developing Nations Student Member Research Grant 2000 of the Animal Behaviour Society (to Sumana) and a CSIR Junior Research Fellowship (to Anindita Bhadra). Behavioural observations were carried out by Sumana with help from Sujata A. Deshpande (13 experiments) and Anindita Bhadra (nine experiments). Data analysis was performed by Anindita Bhadra and Sumana. Anindita Bhadra and Raghavendra Gadagkar co-wrote the paper and Raghavendra Gadagkar supervised the work. Our experiments comply with regulations for animal care in India.

\section{References}

Bhadra A, Iyer PL, Sumana A, Deshpande SA, Ghosh S, Gadagkar R (2007) How do workers of the primitively eusocial wasp Ropalidia marginata detect the presence of their queens? J Theore Biol 246:574-582. doi:10.1016/j.jtbi.2007.01.007

Chandrashekara K, Gadagkar R (1991) Behavioural castes, dominance and division of labour in a primitively eusocial wasp. Ethology 87:269-283

Cuvillier-Hot V, Cobb M, Malosse C, Peeters C (2001) Sex, age and ovarian activity affect cuticular hydrocarbons in Diacamma ceylonense, a queenless ant. J Insect Physiol 47:485-493. doi:10.1016/S0022-1910(00)00137-2

Dani FR, Jones GR, Turillazzi S (1992) Abdomen stroking behaviour and its possible functions in Polistes dominulus (Christ) (Hymenoptera: Vespidae). Behav Proc 28:51-58

Dapporto L, Fondelli L, Turillazzi S (2006) Nestmate recognition and identification of cuticular hydrocarbons composition in the swarm founding paper wasp Ropalidia opifex. Biochem Sys Eco 34:617-625. doi:10.1016/j.bse.2006.04.002

de Biseau J-C, Passera L, Daloze D, Aron S (2004) Ovarian activity correlates with extreme changes in cuticular hydrocarbon profile in the highly polygynous ant Linepithema humile. J Insect Physiol 50:585-593. doi:10.1016/j.jinsphys.2004.04.005 
Deshpande SA, Sumana A, Surbeck M, Gadagkar R (2006) Wasp who would be queen: a comparative study of two primitively eusocial species. Curr Sci 91(3):332-336

D'Ettorre P, Heinze J, Schulz C, Francke W, Ayasse M (2004) Does she smell like a queen? Chemoreception of a cuticular hydrocarbon signal in the ant Pachycondyla inversa. J Exp Biol 207:1085-1091. doi:10.1242/jeb.00865

Dietemann V, Peeters C, Liebig J, Thivet V, Hölldobler B (2003) Cuticular hydrocarbons mediate discrimination of reproductives and nonreproductives in the ant Myrmecia gulosa. Proc Nat Acad Sci USA 100(18):10341-10346. doi:10.1073_pnas.1834281100

Dietemann V, Liebig J, Hölldobler B, Peeters C (2005) Changes in the cuticular hydrocarbons of incipient reproductives correlate with triggering of worker policing in the bulldog ant Myrmecia gulosa. Behav Ecol Sociobiol 58:486-496. doi:10.1007/s00265005-0939-1

Downing HA, Jeanne RL (1985) Communication of status in the social wasp Polistes fuscatus (Hymenoptera: Vespidae). Z Tierpsychol 67:78-96

Endler A, Liebig J, Hölldobler B (2006) Queen fertility, egg marking and colony size in the ant Camponotus floridanus. Behav Ecol Sociobiol 59:490-499. doi:10.1007/s00265-005-0073-0

Fletcher DJC, Ross KG (1985) Regulation of reproduction in eusocial hymenoptera. Ann Rev Entomol 30:319-343

Gadagkar R (2001) The social biology of Ropalidia marginata: toward understanding the evolution of eusociality. Harvard University Press, Cambridge

Gobin B, Billen J, Peeters C (1999) Policing behaviour towards virgin egg layers in a polygynous ponerine ant. Anim Behav 58:11171122

Hannonen M, Sledge MF, Turillazzi S, Sundström L (2002) Queen reproduction, chemical signalling and worker behaviour in polygyne colonies of the ant Formica fusca. Anim Behav 64:477-485. doi:10.1006/anbe.2002.4001

Heinze J, Stengl B, Sledge MF (2002) Worker rank, reproductive status and cuticular hydrocarbon signature in the ant Pachycondyla $c f$. inversa. Behav Ecol Sociobiol 52:59-65. doi:10.1007/s00265-002-0491-1

Kardile SP, Gadagkar R (2002) Docile sitters and active fighters in paper wasps: a tale of two queens. Naturwissenschaften 89:176179. doi:10.1007/s00114-002-0306-2

Klobuchar EA, Deslippe RJ (2002) A queen pheromone induces workers to kill sexual larvae in colonies of the red imported fire ant Solenopsis invicta. Naturwissenschaften 89:302-304. doi:10.1007/s00114-002-0331-1

Liebig J, Peeters C, Hölldobler B (1999) Worker policing limits the number of reproductives in a ponerine ant. Proc R Soc Lond B 266:1865-1870

Liebig J, Peeters C, Oldham NJ, Markstädter C, Hölldobler B (2000) Are variations in cuticular hydrocarbons of queens and workers a reliable signal of fertility in the ant Harpegnathos saltator? Proc Natl Acad Sci USA 97:4124-4131

Monnin T, Malosse C, Peeters C (1998) Solid-phase microextraction and cuticular hydrocarbon differences related to reproductive activity in queenless ant Dinoponera quadriceps. J Chem Ecol 24:473-490. doi:10.1023/A:1022360718870

Premnath S, Sinha A, Gadagkar R (1996) Dominance relationships in the establishment of reproductive division of labour in a primitively eusocial wasp Ropalidia marginata. Behav Ecol Sociobiol 39:125-132. doi:10.1007/s002650050274

Reeve HK (1991) Polistes In: Ross KG, Matthews RW (eds) The social biology of wasps. Cornell University Press, Ithaca, pp 99-148

Sledge MF, Boscaro F, Turillazzi S (2001a) Cuticular hydrocarbons and reproductive status in the social wasp Polistes dominulus. Behav Ecol Sociobiol 49:401-409. doi:10.1007/s002650000311

Sledge MF, Peeters C, Crewe RM (2001b) Reproductive division of labour without dominance interactions in the queenless ponerine ant Pachycondyla (Ophthalmopone) berthoudi. Insectes Sociaux 48:67-73. doi:10.1007/PL00001748

Sumana A, Gadagkar R (2001) The structure of dominance hierarchies in the primitively eusocial wasp Ropalidia marginata. Ethol Ecol Evol 13:273-281

Sumana A, Gadagkar R (2003) Ropalidia marginata-a primitively eusocial wasp society headed by behaviourally non-dominant queens. Curr Sci 84(11):1464-1468

Tibbetts EA (2002) Visual signals of individual identity in the wasp Polistes fuscatus. Proc R Soc Lond B 269:1423-1428. doi:10.1098/rspb.2002.2031

Tibbetts EA, Dale J (2004) A socially enforced signal of quality in a paper wasp. Nature 432:218-222. doi:10.1038/nature02949

Tsuji K, Egashira K, Hölldobler B (1999) Regulation of worker reproduction by direct physical contact in the ant Diacamma sp. from Japan. Anim Behav 58:337-343. doi:10.1006/anbe.1999.1161

Turillazzi S, Sledge MF, Dapporto L, Landi M, Fanelli D, Fondelli L, Zanetti P, Dani FR (2004) Epicuticular lipids and fertility in primitively social wasps (Hymenoptera: Stenogastrinae). Physiol Entomol 29:464-471. doi:10.1111/j.0307-6962.2004.00418.x

Turillazzi S, Dapporto L, Pansolli C, Boulay R, Dani FR, Moneti G, Pieraccini G (2006) Habitually used hibernation sites of paper wasps are marked with venom and cuticular peptides. Curr Biol 16 (14):R530-R531

Van Hooser CA, Gamboa GJ, Fishwild TG (2002) The function of the abdominal stroking in the paper wasp Polistes fuscatus (Hymenoptera: Vespidae). Ethol Ecol Evol 14:141-148

West-Eberhard MJ (1969) The social biology of polistine wasps. Misc Publ Mus Zool Univ Mich 140:1-101

West-Eberhard MJ (1977) The establishment of reproductive dominance in social wasp colonies. In: Proceedings of the 8th international congress international union study of social insects, Wageningen, pp 223-227 